\title{
Aerobic exercise-based cardiac rehabilitation in Chinese patients with coronary heart disease: study protocol for a pilot randomized controlled trial
}

\author{
Richard Y. Cao ${ }^{* \dagger}$ (D), Hongchao Zheng ${ }^{\dagger}$, Qiongyao Mi, Qing Li, Wenchao Yuan, Yueyou Ding and Jian Yang ${ }^{*}$
}

\begin{abstract}
Background: Cardiovascular disease is the leading cause of morbidity and mortality in the world, including China. Cardiac rehabilitation (CR) has been demonstrated to be beneficial in reducing cardiovascular mortality, myocardial infarction, and cerebrovascular events. This pilot study seeks to assess the feasibility of aerobicexercise-based CR in Chinese patients with coronary heart disease (CHD) and outcomes of aerobic metabolism capacity and molecular biomarkers.

Methods/design: This study is a single-center, pilot, randomized, controlled study that is currently being carried out at a regional hospital in Shanghai. Forty patients with CHD who underwent percutaneous coronary intervention will be randomly allocated into either the intervention group or control group. Participants in the intervention group will undergo 8 weeks of aerobic exercise with targeted intensity and participants in the control group will undergo 8 weeks of leisure exercise. The primary measurement is the feasibility of the trial; the secondary measurement is the capacity of aerobic metabolism and the exploratory measurement includes additional molecular biomarkers underlying cardiovascular function.
\end{abstract}

Discussion: This is the first prospective randomized and controlled clinical study in China that assesses the parameters of aerobic metabolism and comprehensively screens for substantial blood biomarkers to reveal the molecular mechanisms underlying changes in cardiovascular function after aerobic exercise with targeted intensity in participants with CHD. The success of this study will contribute to guide the design of future CR studies in patients with CHD in China.

Trial registration: Chinese Clinical Trial Registry, ChiCTR-IPR-17010556. Registered on 1 June 2016.

Keywords: Coronary heart disease, Cardiac rehabilitation, Aerobic metabolism, Biomarker, Protocol, Randomization

\section{Background}

Cardiovascular disease (CVD) is the leading cause of morbidity and mortality in the world [1]. It is not only the top cause of morbidity but also accounts for more than $40 \%$ of all deaths because of the continuous increase in the incidence of CVD due to lifestyle changes, urbanization, and an accelerated rate of aging after rapid economic growth over the past 30 years in China $[2,3]$.

\footnotetext{
* Correspondence: rycao@scrc.ac.cn; jyang@scrc.ac.cn

${ }^{\dagger}$ Richard Y. Cao and Hongchao Zheng contributed equally to this work. The Joint Laboratory of Cardiac Rehabilitation, Shanghai Xuhui Central Hospital \& Shanghai University, 966 Middle Huaihai Road, 200031 \& 99 Shangda Road, Shanghai 200444, China
}

The rising number of CVD deaths in China is largely due to the significant increase in ischemic heart disease, also known as coronary heart disease (CHD), which has been shown to be preventable $[2,4]$. Therefore, prevention and control of CHD in China, a country with almost one fifth of the world's population, may have a significant impact on efforts to achieve sustainable global reductions in CVD rates.

Cardiac rehabilitation (CR), a multifaceted intervention program, has been shown to reduce cardiovascular mortality, myocardial infarction, and cerebrovascular events [5]. It is designed to help patients with CVD achieve optimal physical, psychological, and social status.

(c) The Author(s). 2018 Open Access This article is distributed under the terms of the Creative Commons Attribution 4.0 International License (http://creativecommons.org/licenses/by/4.0/), which permits unrestricted use, distribution, and 
CR helps to stabilize, slow, or even reverse the progression of the underlying atherosclerotic processes, thereby reducing morbidity and mortality $[6,7]$. A large study involving over 600,000 American patients with coronary disease showed that participants undergoing high-dose CR (25 or more sessions) had a lower mortality rate than those undergoing low-dose CR (1-24 sessions) within 5 years of discharge from hospital [8]. CR has been shown to be the third most cost-effective intervention after aspirin and beta blockers to reduce cardiovascular mortality [9]. Moreover, a recent study showed that 3 months of CR, which served as a non-pharmacological way to inhibit platelets, significantly decreased platelet hyperactivity [10].

Although the optimal exercise characteristics (type, dosage, and intensity) that yield the most beneficial effects in patients with CHD are still controversial [11], growing evidence suggests that higher intensity interval training is more effective than lower intensity continuous training for improving aerobic capacity in patients with CHD [12]. This finding is consistent with our previous findings that aerobic exercise with targeted intensity can improve aerobic metabolic capacity in patients who have had a stroke [13]. Markers of aerobic metabolism along with other cardiovascular biomarkers such as $\mathrm{C}$-reactive protein, brain natriuretic peptide, and vascular endothelial growth factor B are important predictors of cardiovascular health or risk [14-16]. Nevertheless, changes in cardiovascular biomarkers after $\mathrm{CR}$ have never been investigated comprehensively.

Here, we designed a pilot, randomized, controlled trial to assess the feasibility of aerobic-exercise-based CR on aerobic metabolic capacity and substantial cardiovascular biomarkers in patients with CHD after percutaneous coronary intervention (PCI). The completion of this pilot study will address aspects of feasibility and key issues of uncertainty to promote future pivotal research in generalizing the CR program in China.

\section{Methods/design}

\section{Aim}

The purpose of this pilot study is to test the feasibility of testing the effect of aerobic-exercise-based CR on aerobic capacity and underlying cardiovascular function expressed by molecular biomarkers in patients with CHD after PCI.

\section{Design}

This pilot study is a single-center, prospective, randomized, and controlled clinical trial that will be carried out in the Department of Rehabilitation, Shanghai Xuhui Central Hospital, between 1 June 2016 and 31 June 2018.
All aspects of the study design and protocol adhere to Standard Protocol Items: Recommendations for Interventional Trials (SPIRIT) guidelines (Additional file 1) [17] and will be reported according to the Consolidated Standards of Reporting Trials (CONSORT) statement (Additional file 2) [18]. The protocol has been reviewed and approved by the Ethics Committee of Shanghai Xuhui Central Hospital (approval number 2016-10). The research team of this study will ensure that the ethics standards are adhered to during the study procedure and that they are in accordance with The Code of Ethics of the World Medical Association (Declaration of Helsinki). Participation will be voluntary, and participants can withdraw from the study at any time without any negative consequences. Written consent forms (Additional file 3) and data collected from participants will be kept anonymous and confidential.

\section{Participant recruitment}

A booklet containing basic information about aerobic-exercise-based CR (Table 1) will be distributed to patients registered for PCI in Shanghai Xuhui Central Hospital. Eligible patients who meet the inclusion criteria will be recruited from the Department of Cardiology at the time of discharge after PCI to participate in the $\mathrm{CR}$ program conducted in the Department of Rehabilitation.

The inclusion criteria are as follows: (1) diagnosis of CHD by a cardiologist based on the combination of clinical symptoms and findings on electrocardiogram and/or echocardiogram and/or coronary angiogram; (2) age between 45 and 80 years; (3) low to moderate risk of cardiovascular event posed by participating in CR based on the Chinese cardiac risk stratification as determined by the experts [19]; and (4) provision of a signed consent form. Strict exclusion criteria are used to avoid unexpected adverse events during rehabilitation training, and these are summarized in Table 2.

\section{Randomization}

Participants will be randomly allocated (1:1 ratio) to either the intervention or control group once screening assessments are completed and eligibility for the study is

Table 1 A booklet containing information on cardiac rehabilitation

List of information related to cardiac rehabilitation

- What cardiac rehabilitation is

- Why patients with cardiovascular diseases need cardiac rehabilitation

- What cardiopulmonary exercise testing is

-Who can take part in the aerobic exercise-based cardiac rehabilitation

- What individualized exercise prescription is

- A brief introduction to the Department of Rehabilitation in Shanghai Xuhui Central Hospital 
Table 2 Exclusion criteria for the cardiac rehabilitation program

- Cognitive impairment or mental disorder identified by MMSE score < 24

- Unstable angina or onset of myocardial infarction within $<2$ weeks

- Uncontrolled serious arrhythmia

- Uncontrolled hypertension (resting systolic blood pressure > $160 \mathrm{mmHg}$ or resting diastolic blood pressure $>100 \mathrm{mmHg}$ )

- Cardiac function class IV

- Coronary heart disease accompanied by acute complications (coronary artery dissection, ventricular aneurysm, large area of myocardial infarction associated with shock, acute vascular occlusion including stent thrombosis) and high risk of cardiovascular events posed by rehabilitation exercise

- Active pericarditis or myocarditis, serious infection, chronic obstructive pulmonary disease, moderate to serious aortic stenosis, resting heart rate after drug control > 100 times/min

- New deep vein thrombosis, thrombophlebitis, aortic dissection or aneurysm in other parts of the body, lower limb occlusive atherosclerosis

- Inability to tolerate exercise due to fracture, arthritis or muscle pain

- Abnormal electrolytes such as potassium, sodium or calcium

- Uncontrolled hyperthyroidism or hypothyroidism at the time of recruitment

- COPD with signs of infection such as fever, sore throat, coughing, etc. COPD chronic obstructive pulmonary disease, MMSE mini-mental state examination

confirmed (Fig. 1). A research coordinator will generate the random allocation sequence, enroll participants, and assign participants to interventions. A stratified randomization scheme based on gender will be used. Microsoft Excel Formula Rand will be used to allocate patients to the intervention or control group. A random number between 0 and 1 will be generated for each patient; if the last digit is an even number the patient will be assigned to group A (intervention) and if the last digit is an odd number the patient will be assigned to group B (control) (Table 3). Forty participants will be allocated randomly to group A (intervention exercise group with targeted intensity, $n=20$ ) or $\mathrm{B}$ (control group with leisure exercise, $n=20$ ).

\section{Sample size determination}

Given the pilot nature of this trial, we will focus on the feasibility of the study and ensure that the exercise intervention runs smoothly, we will not calculate a sample size to determine the power in this study [20]. However, it is recommended that a minimum of 30 participants is required to achieve sufficient precision to enable sample size calculation for subsequent studies [21]. Therefore, we plan to recruit 40 participants, which will allow the completion of data collection from 30 subjects, with a $75 \%$ participant retention rate.

\section{Study procedures}

Telephone follow up before exercise intervention

Eligible patients who are willing to participate in our CR program will be contacted by a telephone call $1-2$ weeks after PCI. Patients in a stable condition will be recommended to start outpatient rehabilitation in our clinic as soon as possible. A "stable condition" is determined by the following: the participant self-reports satisfaction over the phone and denies recent chest pain or any other disorders that need additional medications; the physician confirms that the patient's vital signs are stable and within normal limits at the outpatient clinic, for example, resting heart rate $<100 / \mathrm{min}$, resting systolic blood pressure $<140 \mathrm{mmHg}$ and diastolic blood pressure $<90 \mathrm{mmHg}$. If an individual is not considered stable, there is an additional waiting period of 2 weeks. That person will be checked again 2 weeks later to determine if he/she is sufficiently stable to participate in the CR program.

\section{Exercise prescription}

All participants will undergo cardiopulmonary exercise testing (CPET) to obtain initial parameters of anaerobic threshold (AnT), which is defined as the highest sustained intensity of exercise for which measurement of oxygen uptake by means of CPET can account for the entire energy requirement, on the first day of the CR program. The targeted intensity is determined based on the heart rate recorded 1 min before AnT during CPET to provide an individualized $\mathrm{CR}$ training prescription for each participant in the intervention group. Thus, exercise intensity will not be affected by medications because the intensity is determined on an individual basis. Participants in the control group will undergo the same number of exercise sessions without targeted intensity. The $\mathrm{CR}$ program also includes CHD management strategies such as concomitant therapy, balanced diet nutrition, psychosocial consultation, and smoking cessation. There are no differences in disease management between the two groups.

\section{Exercise intervention}

The exercise intervention is an outpatient clinical rehabilitation program delivered one-on-one to individuals in a 30 -min session and three times a week for 8 weeks in the hospital outpatient clinic. The 30 -min CR procedure includes $5 \mathrm{~min}$ of warm-up exercise, $20 \mathrm{~min}$ of cycle ergometer (Ergoline GmbH, Germany) exercise with targeted intensity, and $5 \mathrm{~min}$ of cool-down exercise. The targeted intensity is set as the heart rate $1 \mathrm{~min}$ before AnT based on CPET. We expect that exercise parameters will improve gradually as individuals reach their targeted intensity more frequently with time over the 8 weeks. Participants are closely monitored using a heart rate monitoring device connected to the cycle ergometer and will be guided by the CR team, which consists of cardiologists, rehabilitation physicians, physiotherapists, nurses, and clinical research coordinators. The research coordinator will record the attendance of each participant and will ask the participant to come on the 


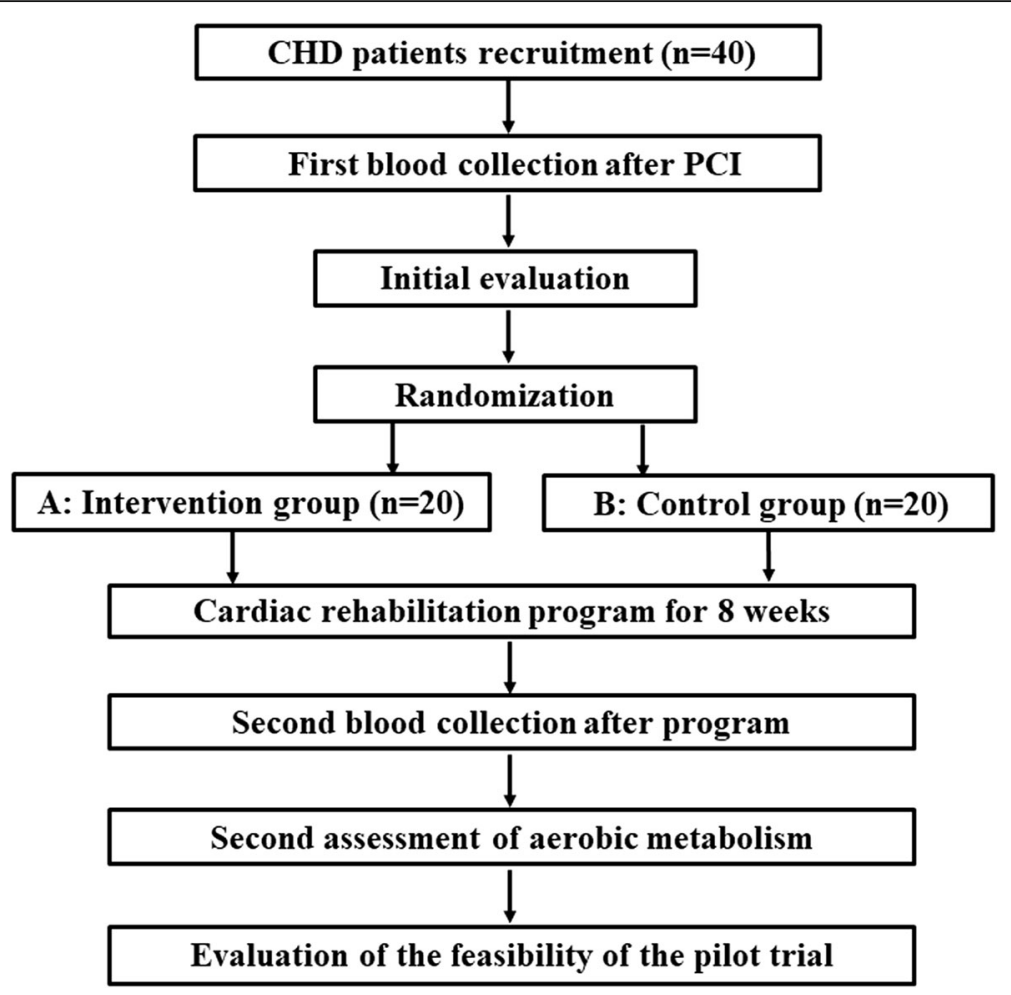

Fig. 1 Study flow chart. Eligible participants with coronary heart disease (CHD) after percutaneous coronary intervention (PCI) will be randomly allocated to the intervention or control group. The exercise intervention is an outpatient clinical rehabilitation program with targeted intensity, which includes a 30-min session three times a week for 8 weeks. Participants in the control group will be prescribed the same number of exercise sessions without targeted intensity. Cardiopulmonary exercise testing will be performed before and after the 8-week cardiac rehabilitation program to assess changes in cardiopulmonary function

weekend to compensate for any missed sessions. In addition, the CR protocol can be extended to accommodate an intervening illness. Nevertheless, the participant is allowed to quit the program at any time if he/she wants to do so. Participants will be followed closely to ensure adherence. All CR providers are trained to follow the same protocol to ensure intervention fidelity. Participants in the control arm will receive telephone calls once a week to monitor their physical conditions and will be encouraged to take a walk at least $30 \mathrm{~min}$ at a time and three times a week during the 8-week study at home but not at the program center. The information for the control group will be recorded using activity tracking apps such as activity tracker bands, watches and smartphones so that the authors will know how active the control-arm participants are. The second CPET will be conducted after the 8-week CR program. Graduates will also be advised to take concomitant medications, continue engaging in appropriate exercise, eat a balanced diet, and quit smoking.

\section{Telephone follow up after 3 months}

Participants in both groups will receive follow-up calls 3 months after the end of the program to provide information on their most updated health condition such as smoking status, cardiac risk control medication (aspirin, statins, beta-blockers, etc.), diet, psychological status, and physical activity. At the end of the CR session, patients in both the intervention and control groups will be prescribed a home exercise program using community-based rehabilitation facilities and will be encouraged to live a more active lifestyle.

\section{Data collection}

Assessors are trained to collect trial data from the outpatient clinic and laboratory. The following data will be collected from all participants before and after the rehabilitation training as shown in the SPIRIT diagram (Fig. 2): the aerobic metabolism parameter (AnT), clinical indicators (blood pressure, heart rate, body mass index, smoking status, and comorbidities), laboratory indicators (total cholesterol, high-density lipids (HDL)-cholesterol, low-density lipids (LDL)-cholesterol, triacylglycerides, apolipoproteins, homocysteine, glucose, etc.), and blood samples for additional biomarkers (C-reactive protein, macrophage inflammatory protein- $1 \alpha$, pentraxin-3, monocyte chemotactic protein-1, interleukins, transforming growth factor- $\beta$, interferon- $\gamma$, brain natriuretic peptide, growth differentiation factor-15, vascular endothelial 
Table 3 A stratified randomization scheme based on gender

\begin{tabular}{|c|c|c|c|c|c|}
\hline \multicolumn{3}{|l|}{ Male } & \multicolumn{3}{|l|}{ Female } \\
\hline $\begin{array}{l}\text { Serial } \\
\text { number }\end{array}$ & $\begin{array}{l}\text { Random } \\
\text { number }\end{array}$ & Group & $\begin{array}{l}\text { Serial } \\
\text { number }\end{array}$ & $\begin{array}{l}\text { Random } \\
\text { number }\end{array}$ & $\overline{\text { Group }}$ \\
\hline 1 & 0.699718 & A & 1 & 0.299478 & $A$ \\
\hline 2 & 0.474505 & B & 2 & 0.228082 & A \\
\hline 3 & 0.278238 & A & 3 & 0.905825 & B \\
\hline 4 & 0.063581 & B & 4 & 0.288985 & B \\
\hline 5 & 0.543526 & A & 5 & 0.204267 & B \\
\hline 6 & 0.645542 & A & 6 & 0.004536 & A \\
\hline 7 & 0.606382 & A & 7 & 0.876704 & A \\
\hline 8 & 0.358782 & A & 8 & 0.765598 & A \\
\hline 9 & 0.913533 & B & 9 & 0.989875 & B \\
\hline 10 & 0.394207 & B & 10 & 0.735247 & B \\
\hline 11 & 0.101518 & A & 11 & 0.968638 & A \\
\hline 12 & 0.830019 & B & 12 & 0.975038 & A \\
\hline 13 & 0.454501 & B & 13 & 0.355564 & A \\
\hline 14 & 0.941391 & B & 14 & 0.730377 & B \\
\hline 15 & 0.748873 & B & 15 & 0.087323 & B \\
\hline 16 & 0.736089 & B & 16 & 0.318215 & B \\
\hline 17 & 0.026114 & A & 17 & 0.794593 & B \\
\hline 18 & 0.294124 & A & 18 & 0.322396 & A \\
\hline 19 & 0.801002 & A & 19 & 0.180908 & A \\
\hline 20 & 0.448855 & B & 20 & 0.552161 & B \\
\hline
\end{tabular}

Microsoft Excel Rand is performed to generate random numbers between 0 and 1, last digit even number for group A (intervention) and odd for group B (control). Patients are then allocated to intervention and control groups chronologically

growth factor-B, myeloperoxidase, adiponectin, matrix metalloproteinase-1, 2, or 9, matrix metalloproteinases tissue inhibitor-1, phosphodiesterase-9A). Data will be collected at 3 months to assess the short-term prognosis and risk factors for cardiac adverse events after the CR program.

\section{Outcome measurements}

The primary outcome is the feasibility of the trial. Recruitment rate and retention rate as feasibility parameters will be analyzed. The secondary outcomes are aerobic metabolism parameters and clinical and laboratory indicators that will be used to identify cardiac risk factors such as hypertension, dyslipidemia, diabetes mellitus, obesity, and smoking status. The exploratory outcomes are additional biomarkers such as inflammatory markers (C-reactive protein, macrophage inflammatory protein- $1 \alpha$, pentraxin- 3 , monocyte chemotactic protein-1, interleukins, transforming growth factor $\beta$ ), an immune-modulatory marker (interferon- $\gamma$ ), myocyte stress markers (brain natriuretic peptide and growth differentiation factor-15), a left ventricular dysfunction marker (vascular endothelial growth factor-B), an oxidative stress marker (myeloperoxidase), a metabolic hormone (adiponectin), extracellular-matrix remodeling markers (matrix metalloproteinase-1, 2, 9, and matrix metalloproteinases tissue inhibitor-1), and a signal transduction regulator (phosphodiesterase-9A) to further evaluate a patient's underlying cardiovascular function at the molecular level.

\section{Data assessment}

The objective of this pilot study is to test the feasibility of recruitment, retention, intervention, and collection of data on aerobic metabolism parameters and clinical and laboratory measurements at the same time. Mean changes in outcomes over time will be summarized graphically and descriptively within each group. These changes will be compared between groups and will be described with associated 95\% confidence intervals to explore possible effects of the intervention, recognizing that this pilot trial is not powered to detect clinically meaningful effects. Feasibility parameters, such as recruitment rate and retention rate, will be analyzed to determine whether it is worth continuing to conduct a large-scale trial that builds from the current pilot trial. The key feasibility criteria include recruitment of 40 participants, attainment of a $75 \%$ participant retention rate, and measurement of all outcomes in $90 \%$ of all participants. These parameters will be assessed at the end of the pilot trial by a statistician who is not involved in the study.

\section{Discussion}

The benefits of CR are well-recognized in America and Europe and are widely recommended by the American Association of Cardiovascular and Pulmonary Rehabilitation and European Society of Cardiology as secondary prevention in patients with coronary disease [7, 22, 23]. However, the CR program is far underdeveloped and in China has thereby resulted in a high rate of recurrence of heart attacks and adverse cardiovascular events [24]. China faces an aging population with an increasing lifespan, which will result in a large proportion of people suffering from cardiovascular events in the near future. Therefore, increasing awareness of the benefits of CR, improving $\mathrm{CR}$ availability throughout China, and providing safe and effective CR programs for Chinese patients with $\mathrm{CHD}$ are extremely important.

To our knowledge, the present pilot trial is the first clinical study that comprehensively screens multiple blood biomarkers to explore the potential underlying molecular mechanisms and changes in cardiovascular function after aerobic exercise-based CR training. Results of this pilot study can be used to predict the feasibility and operational acceptability of a future large-scale pivotal trial that promotes the generalization of the CR program in China. 


\begin{tabular}{|c|c|c|c|c|c|}
\hline \multirow[b]{3}{*}{ TIME POINT } & \multicolumn{5}{|c|}{ STUDY PERIOD } \\
\hline & \multirow{2}{*}{\begin{tabular}{|c|} 
Enrollment \\
$\mathrm{PCl}$ discharge \\
\end{tabular}} & \multirow{2}{*}{$\begin{array}{c}\text { Allocation } \\
\text { Week } 0\end{array}$} & \multirow{2}{*}{\begin{tabular}{|c|} 
Post-allocation \\
Week 1-8 \\
\end{tabular}} & \multicolumn{2}{|c|}{ Close-out } \\
\hline & & & & Week 9 & after 3 months \\
\hline Eligibility screening & $x$ & & & & \\
\hline Informed consent & $x$ & & & & \\
\hline First blood collection & & $x$ & & & \\
\hline Initial assessment & & $x$ & & & \\
\hline Allocation & & $x$ & & & \\
\hline \multicolumn{6}{|l|}{ Exercise Interventions } \\
\hline Group A: intensive exercise & & & $x$ & & \\
\hline Group B: leisure exercise & & & $x$ & & \\
\hline Second blood collection & & & & $x$ & \\
\hline \multicolumn{6}{|l|}{ Second assessment } \\
\hline Aerobic metabolism & & & & $x$ & \\
\hline Clinical indicators & & & & $x$ & \\
\hline ELISA biomarkers & & & & $x$ & \\
\hline Telephone follow-up & & & & & $x$ \\
\hline
\end{tabular}

Fig. 2 Standard Protocol Items: Recommendations for Interventional Trials (SPIRIT) timeline of measurements. Recommended content for the schedule of enrollment, intervention, and assessments. ELISA, enzyme linked immunosorbent assay; PCI, percutaneous coronary intervention

However, this pilot trial has several limitations. Given the large age range of subjects from 45 to 80 years and the relatively small sample size after allocation to subgroups (male and female), physiology and biomarker profiles can vary greatly. The second limitation is the disparate modes between the intervention (cycle ergometer) and control (walk) groups. Specifically, the intervention group receives cycle ergometer training for exercise safety and to prevent patients from falling during treadmill training whereas the control arm does not receive this training. Moreover, whereas all of the intervention participants will be monitored with a standardized heart rate monitor during each intervention session, activity in the control arm will not be monitored using the same tool and will instead be monitored by whatever tracking app the participant has available. As a consequence, the data quality may be of very low quality or missing in the control arm. The third limitation is that outcome assessment of metabolism and clinical indicators (blood pressure, heart rate etc.) is not blinded. As a consequence, the quantitative results of the trial may not be completely reliable as there could be different quality of assessments in the two groups of the trial. However, a statistician who is not involved in the study will be invited to assess the data according to a pre-specified analysis plan, and the groups will be labeled A and $\mathrm{B}$, to ensure that the data are analyzed blind. In a future larger trial, procedures will be put in place to ensure blinding of outcome assessors to avoid bias. The feasibility measures are the focus of the current pilot study and these will not be subject to potential observer bias.

\section{Trial status}

This is an ongoing trial. We have not completed patient recruitment at the time of submission. So far, 13 patients have been enrolled, with 6 in the intervention group and 7 in the control group.

\section{Additional files}

Additional file 1: SPIRIT checklist. (DOCX $38 \mathrm{~kb})$

Additional file 2: CONSORT checklist. (DOC $226 \mathrm{~kb}$ )

Additional file 3: Informed consent. (DOCX 26 kb)

\section{Abbreviations}

AnT: Anaerobic threshold; CHD: Coronary heart disease; CONSORT: Consolidated Standards of Reporting Trials;

CPET: Cardiopulmonary exercise testing; CR: Cardiac rehabilitation;

CVD: Cardiovascular disease; PCl: Percutaneous coronary intervention;

SPIRIT: Standard Protocol Items: Recommendations for Interventional Trials

\section{Acknowledgements}

The authors would like to thank Li Fan, Gongliang Liu, and Weidong Ni from the Department of Rehabilitation, Shanghai Xuhui Central Hospital for their generous assistance in this study and Nature Research Editing Service for English editing.

\section{Funding}

This work is supported by Shanghai Municipal Commission of Health and Family Planning (JY: ZK2015A29 and HZ: ZK2015A30) and National Natural Science Foundation of China (RYC: 81672260).

Availability of data and materials

Supporting data such as ethical, consent, and funding documents are available for review by the Editorial Office. 


\section{Authors' contributions}

RYC, JY, and HZ conceived, designed, and coordinated this study. RYC and QM drafted the manuscript and developed the randomization scheme. QL participated in the study design and coordinated the study. WY and YD assisted in coordinating the study. RYC, HZ, and JY helped to edit the manuscript. All authors have read and approved the final manuscript.

\section{Ethics approval and consent to participate}

Ethics approval of the study design and protocol has been given by the Ethics Committee of Shanghai Xuhui Central Hospital (approval number 2016-10). Participation will be voluntary, and participants can withdraw from the study at any time without any negative consequences. Written informed consent will be obtained from all participants before the initial CPET.

\section{Consent for publication}

Not applicable.

\section{Competing interests}

The authors declare that they have no competing interests.

\section{Publisher's Note}

Springer Nature remains neutral with regard to jurisdictional claims in published maps and institutional affiliations.

Received: 22 May 2017 Accepted: 28 June 2018

Published online: 09 July 2018

\section{References}

1. GBD 2013 Mortality and Causes of Death Collaborators. Global, regional, and national age sex specific all-cause and cause-specific mortality for 240 causes of death, 1990-2013: a systematic analysis for the Global Burden of Disease Study 2013. Lancet. 2015;385:117-71.

2. Chen WW, Gao RL, Liu LS, Zhu ML, Wang W, Wang YJ, et al. China cardiovascular diseases report 2015: a summary. J Geriatr Cardiol. 2017;14:1-10.

3. Wu Y, Benjamin EJ, Macmahon S. Prevention and control of cardiovascular disease in the rapidly changing economy of China. Circulation. 2016;133:2545.

4. Wong ND. Epidemiological studies of CHD and the evolution of preventive cardiology. Nat Rev Cardiol. 2014;11:276-89.

5. van Halewijn G, Deckers J, Tay HY, van Domburg R, Kotseva K, Wood D. Lessons from contemporary trials of cardiovascular prevention and rehabilitation: a systematic review and meta-analysis. Int J Cardiol. 2017;232:294-303.

6. Lavie CJ, Arena R, Swift DL, Johannsen NM, Sui X, Lee DC, et al. Exercise and the cardiovascular system: clinical science and cardiovascular outcomes. Circ Res. 2015:117:207-19.

7. American Association of Cardiovascular and Pulmonary Rehabilitation; American College of Cardiology Foundation; American Heart Association Task Force on Performance Measures (Writing Committee to Develop Clinical Performance Measures for Cardiac Rehabilitation), Thomas RJ, King M, Lui K, Oldridge N, Piña IL, Spertus J. AACVPR/ACCF/AHA 2010 update: performance measures on cardiac rehabilitation for referral to cardiac rehabilitation/secondary prevention services endorsed by the American College of Chest Physicians, the American College of Sports Medicine, the American Physical Therapy Association, the Canadian Association of Cardiac Rehabilitation, the Clinical Exercise Physiology Association, the European Association for Cardiovascular Prevention and Rehabilitation, the InterAmerican Heart Foundation, the National Association of Clinical Nurse Specialists, the Preventive Cardiovascular Nurses Association, and the Society of Thoracic Surgeons. J Am Coll Cardiol. 2010;56:1159-67.

8. Suaya JA, Stason WB, Ades PA, Normand SL, Shepard DS. Cardiac rehabilitation and survival in older coronary patients. J Am Coll Cardiol. 2009:54:25-33.

9. Fidan D, Unal B, Critchley J, Capewell S. Economic analysis of treatments reducing coronary heart disease mortality in England and Wales, 20002010. OJM. 2007:100:277-89.

10. Tóth-Zsámboki E, Horváth Z, Hajtman L, Leé S, Pállinger É, Kuklis E, Tahy Á et al. Cardiac rehabilitation programme as a non-pharmacological platelet inhibitory tool in acute coronary syndrome survivors. Eur J Prev Cardiol. 2017; doi.org/10.1177/2047487317704937

11. Vanhees L, Rauch B, Piepoli M, van Buuren F, Takken T, Börjesson M, et al. Importance of characteristics and modalities of physical activity and exercise in the management of cardiovascular health in individuals with cardiovascular risk factors: recommendations from the EACPR. Part II. Eur J Prev Cardiol. 2012:19:1005-33.

12. Elliott AD, Rajopadhyaya K, Bentley DJ, Beltrame JF, Aromataris EC. Interval training versus continuous exercise in patients with coronary artery disease: a meta-analysis. Heart Lung Circ. 2015;24:149-57.

13. Li Q, Yang J, Li F, Yuan W, Fangling W, Zhang Y, et al. Effects of monitoring aerobic exercise with target intensity on the aerobic metabolism capacity and physical indicators in stroke patients which combined with coronary artery disease. Chinese J Rehabil Med. 2016;31:183-8. Article in Chinese

14. Cao RY, Zheng H, Guo J, Redfearn DP. Prognostic value of plasma biomarkers in patients with acute coronary syndrome: a review of advances in the past decade. Biomark Med. 2016;10:525-35.

15. van Kimmenade RR, Januzzi JL Jr. Emerging biomarkers in heart failure. Clin Chem. 2012;58:127-38

16. Braunwald E. Biomarkers in heart failure. N Engl J Med. 2008:358:2148-59.

17. Li T, Boutron I, Salman RA, Cobo E, Flemyng E, Grimshaw JM, et al. Review and publication of protocol submissions to trials - what have we learned in 10 years? Trials. 2017;18:1-5.

18. Eldridge SM, Chan CL, Campbell MJ, Bond CM, Hopewell S, Thabane L, et al. CONSORT 2010 statement: extension to randomised pilot and feasibility trials. BMJ. 2016;355:i5239.

19. Chinese Society of Cardiology of Chinese Medical Association; Cardiovascular Committee of Chinese Association of Rehabilitation Medicine; Committee of Cardio-Cerebral-Vascular Diseases of Gerontological Society of China. Chinese expert's consensus on cardiac rehabilitation/ secondary prevention for coronary artery disease. Zhonghua Xin Xue Guan Bing Za Zhi. 2013;41:267-75.

20. Lancaster GA. Pilot and feasibility studies come of age. Pilot Feas Stud. 2015;1:1-4.

21. Lancaster GA, Dodd S, Williamson PR. Design and analysis of pilot studies: recommendations for good practice. J Eval Clin Pract. 2004;10(2):307-12.

22. Piepoli MF, Corrà U, Adamopoulos S, Benzer W, Bjarnason-Wehrens B, Cupples $\mathrm{M}$, et al. Secondary prevention in the clinical management of patients with cardiovascular diseases. Core components, standards and outcome measures for referral and delivery: a policy statement from the cardiac rehabilitation section of the European Association for Cardiovascular Prevention \& Rehabilitation Endorsed by the Committee for Practice Guidelines of the European Society of Cardiology. Eur J Prev Cardiol. 2014;21:664-81.

23. Riley RF, Don CW, Aldea GS, Mokadam NA, Probstfield J, Maynard C, et al. Recent trends in adherence to secondary prevention guidelines for patients undergoing coronary revascularization in Washington state: an analysis of the clinical outcomes assessment program (COAP) registry. J Am Heart Assoc. 2012; https://doi.org/10.1161/JAHA.112.002733.

24. Wang W, Chair SY, Thompson DR, Twinn SF. Health care professionals perceptions of hospital-based cardiac rehabilitation in mainland China: an exploratory study. J Clin Nurs. 2009;18:3401-8.

\section{Ready to submit your research? Choose BMC and benefit from:}

- fast, convenient online submission

- thorough peer review by experienced researchers in your field

- rapid publication on acceptance

- support for research data, including large and complex data types

- gold Open Access which fosters wider collaboration and increased citations

- maximum visibility for your research: over $100 \mathrm{M}$ website views per year

At BMC, research is always in progress.

Learn more biomedcentral.com/submissions 